\title{
Multilinguales
}

\section{De Tunis à Kairouan de Guy de Maupassant. Voyage au bout des origines}

$$
\text { "من تونس الى القيروان"غي دي موبسان ورحلة البحث في الأصول }
$$

\section{Dorra Barhoumi}

\section{(2) OpenEdition}

\section{Journals}

Édition électronique

URL : http://journals.openedition.org/multilinguales/301

DOI : $10.4000 /$ multilinguales.301

ISSN : 2335-1853

Éditeur

Université Abderrahmane Mira - Bejaia

Référence électronique

Dorra Barhoumi, « De Tunis à Kairouan de Guy de Maupassant. Voyage au bout des origines »,

Multilinguales [En ligne], 8 | 2017, mis en ligne le 01 juin 2017, consulté le 10 décembre 2020. URL:

http://journals.openedition.org/multilinguales/301 ; DOI : https://doi.org/10.4000/multilinguales.301

Ce document a été généré automatiquement le 10 décembre 2020.

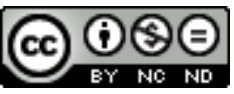

Multilinguales est mise à disposition selon les termes de la Licence Creative Commons Attribution -

Pas d'Utilisation Commerciale - Pas de Modification 4.0 International 


\title{
De Tunis à Kairouan de Guy de Maupassant. Voyage au bout des origines
}

\author{
"من تونس الى القيروان"غي دي موبسان ورحلة البحث في الأصول
}

\section{Dorra Barhoumi}

1 Maupassant ne cesse de critiquer, souvent avec virulence, et constamment avec un réalisme amer, ses origines d'abord d'homme voué à la souffrance existentielle, d'Européen civilisé ensuite et surtout son appartenance à la société française, orgueilleuse. Les contes normands, à titre d'exemple, racontent souvent des histoires cruelles qui révèlent l'égoïsme, l'insensibilité et l'âpreté au gain. Dans Notre cœur ou BelAmi, Maupassant peint l'absurdité et la médiocrité aussi bien des paysans de sa Normandie natale que de la bourgeoisie parisienne ${ }^{1}$. Par ailleurs, il évoque dans ses contes et nouvelles, l'animosité de l'homme, la dégradation des valeurs authentiques au profit du matérialisme. Avec acharnement souvent, Maupassant lutte contre la répétition et l'automatisme de la même action monotone d'un milieu qui ne se lasse de revivre la même chose sur le même rythme.

Après une éblouissante fertilité littéraire et à l'âge de trente sept ans (cinq ans avant sa mort), Maupassant excédé et épuisé, aspire au repos. Il le cherche alors, selon Yvan Leclerc, dans «la chaleur d'Afrique pour soigner un dérèglement nerveux qu'il attribue aux brouillards $d u$ Nord. $»^{2}$. Après tant de voyages en Angleterre, en Corse, en Italie, en Cécilie, en Provence, etc., l'envie de visiter l'Afrique hante Maupassant pour en redécouvrir cette fois-ci, corps et âme, le charme des origines de ce continent inconnu. Maupassant a déjà découvert à travers le roman Salammbô de son maitre et ami Gustave Flaubert, l'Afrique du Nord et plus précisément la Tunisie par l'observation et la réflexion "d'une situation d'écriture romanesque (...) sur les lieux des actions de son roman carthaginois. » (Mittérand, 1993 : 9). Mais la curiosité de l'homme de Lettres le pousse à vérifier par de propres yeux et donc de voir de près et par tout son être, les trois villes phares : Alger, Tunis et Kairouan, les portes de l'Afrique mystérieuse. 
3 La chronique De Tunis à Kairouan (Guy de Maupassant, 1993) était publiée en plusieurs parties dans «Le Gaulois » du 11 décembre 1888 et dans « La Revue des Deux Mondes » en 1889 (Delaisement, 2004), et relatant un voyage que Maupassant a effectué dans les deux colonies maghrébines, l'Algérie et surtout la Tunisie. Dans ce récit, Maupassant s'évade et erre pour oublier son mal et pour rompre, ne serait-ce pour une courte durée, avec son lourd héritage. Il tente alors de « se déraciner » en explorant une terre inconnue et des origines vierges de tout sens artificiel, d'absurdité et de férocité propres à son monde. Maupassant se dirige vers un univers où tout est différent, naturel, primitif, simple, farouche, ethnique, culturel, historique, religieux, mystique, fanatique, profane, vertueux, authentique, paradoxal, divers, mystérieux, fataliste, beau et fascinant.

4 La problématique de notre réflexion s'articule alors sur le récit de voyage De Tunis à Kairouan et sur la découverte de Maupassant non seulement en tant que voyageur connaisseur qui entrait en Algérie et en Tunisie par les écrits de Flaubert, mais en tant que curieux qui cherchait à s'introduire dans les petits détails des origines d'un peuple pour découvrir le brassage de sa culture, son héritage et tout un univers propre à lui. Ainsi, l'hypothèse de notre article est de comprendre la position de Maupassant à travers sa fascination devant ces origines africaines, berbères, arabes, musulmanes, juives et chrétiennes. Aussi, sur la comparaison à laquelle procède Maupassant à travers ces récits de voyages, entre ces origines nord-africaines et ses propres origines européennes.

Maupassant écrit dans son journal de voyage : «La vie si courte, si longue, devient parfois insupportable. Elle se déroule, toujours pareille, avec la mort au bout. (...) Alors on se sent écrasé sous le sentiment de l'éternelle misère de tout, de l'impuissance humaine et de la monotonie des actions. » (3). Aussi, Ainsi commence le premier volet des chroniques du voyageur. Le ton de ces premières phrases est dominé par le dégoût d'une vie itérative, le désespoir d'une existence étrange, et l'absurdité d'un monde illusoire et fallacieux (Bury, 1988). L'écrivain cherche un dépaysement et un changement renversants lui apportant l'authenticité qu'il cherchait à explorer dans l'ailleurs et lui permettant de vivre l'exotisme tant rêvé à l'intérieur. Las de ses précédents voyages européens, suffoqué par son entourage normand ainsi que par son environnement parisien, l'écrivain cherche une autre source d'inspiration qui apaiserait son âme désœuvrée du spectacle répétitif qu'il vivait continuellement chez lui en France. Il disait à ce propos :

Quand on est las, las à pleurer du matin au soir, las à ne plus avoir la force de se lever pour boire un verre d'eau, las des visages amis vus trop souvent et devenus irritants, des odieux et placides voisins, des choses familières et monotones, de sa maison, de sa rue, de sa bonne, (...) de son chien, des taches immuables des tentures, de la régularités des repas, du sommeil dans le même lit, de chaque action répétée chaque jour, las de soi-même, de sa propre voix (...), il faut partir, entrer dans une vie nouvelle et changeante .(4)

6 Accablé, dégoûté et exténué, Maupassant écrit sa misère d'exister dans un monde dominé par la platitude, et décrit les douleurs de ses racines asphyxiées du poids de l'absurdité et de la facticité de sa vie sociale. Il souhaite mettre fin à sa souffrance d'un homme trop conscient, ne serait-ce par la magie du dépaysement. Il faut donc «Fuir, partir! Fuir les lieux connus, les hommes, les mouvements pareils aux mêmes heures, et les mêmes pensées surtout. (...) Le voyage est une espèce de porte où l'ont sort de la réalité connue pour pénétrer dans une réalité inexplorée qui semble un rêve. " (3-4). Voyager, donc, non pas 
en tant que colonisateur qui exploite injustement la terre du colonisé, mais en tant que rêveur en quête du salut, du vrai bonheur (Tverdota, 1994). Couper les veines du mal afin de se purifier, corps et âme. Retrouver, peut-être enfin, les véritables origines oniriques. Maupassant choisit l'Afrique par «un impérieux besoin, par la nostalgie du Désert ignoré, comme par pressentiment d'une passion qui va naître. » (5).

Ce Normand passionné et cet écrivain passionnel vivait depuis une période une panne d'inspiration et part en quête d'une nouvelle et inhabituelle «Passion", sa muse et son eau de vie. Il se dirige donc vers un lointain endroit contenant les mêmes éléments naturels qui le font vivre et auxquels il est intimement et héréditairement attaché : l'eau, la mer, les ports, les navires, la lumière. Maupassant repart en 1887 pour la deuxième fois en Afrique via Marseille, après un voyage effectué en 1881 sous la conduite de Jules Lemaître, à Alger, à Oran, au sud d'Algérie, à Constantine et en Kabylie. Son deuxième grand voyage commence par Alger en allant à Tunis puis à Kairouan et enfin à Sousse. Son parcours s'arrête à Tunis et son voyage s'achève en 1888.

8 L'Algérie était le premier pays à redécouvrir. Sur les quais d'Alger et les ruelles des villes dites «indigènes", sur ses montagnes côtières ou dans les dunes et sables du Sahara, c'était la même image que Maupassant croisait souvent, celle d'une figure mystique, dure et fière. C'est la même allure qu'il observait à travers "tous ces corps drapés comme en des robes de moines, la tête encapuchonnée sous le turban flottant parderrière.» (7). Ce qui a fasciné, par ailleurs, le regard de Maupassant c'est surtout les attitudes cyniques de ces hommes qui ressemblent aux " prêtres » dans leur démarche, à des « apôtres pêcheurs » dans leurs gestes et à des «mystiques » dans leurs attitudes méprisantes et hostiles au monde. Il décrit ce caractère unique de ces hommes en leur attachement solide à la religion musulmane car :

La religion, écrit Maupassant, est la grande inspiratrice de leurs actes, de leur âme, de leurs qualités et de leurs défauts. (...)Nous ne découvrons guère la nature spontanée ou primitive de l'Arabe sans qu'elle ait été, pour ainsi dire, recréée par sa croyance, par le Coran, par l'enseignement de Mohammed. Jamais aucune autre religion ne s'est incarnée ainsi en ces êtres. (8)

9 L'Arabe apparaît chez Maupassant tel un ensemble de caractères, de tendances et d'attitudes qui forme un type et une entité humaine et naturelle à part entière. L'Arabe avec un grand 'A' connote la grandeur de sa nature humaine propre à lui seul et inégalable aux autres. A Alger, Maupassant est subjugué par le cadre religieux dans lequel évoluent ces Arabes trop attachés à leur foi et entame sa description par «la mosquée blanche » au « bout du quai d'Alger »:

sans cesse, les Arabes entrent, des humbles, des riches, le portefaix du port et l'ancien chef, le noble sous la blancheur soyeuse de son burnous éclatant. Tous, pieds nus, font les mêmes gestes, prient le même Dieu avec la même foi exaltée et simple, sans pause et sans distraction. (10)

10 Par ailleurs, Maupassant décrit les femmes musulmanes et leur particularité dans leur attachement religieux aux marabouts. Car selon son observation, les femmes ont le droit d'entrer à la mosquée mais « elles ne viennent presque jamais » car « Dieu est trop loin, trop haut, trop imposant pour elles.» (11). La zaouïa Abde-er-Rahman-el-Taalbi est celle qui a le plus fasciné Maupassant à Alger, parce qu'il trouvait en elle tout un ensemble d'institutions : le cimetière du marabout, une annexe de la mosquée, une école et même un cours de hauts enseignements pour les musulmans lettrés. C'est alors ici que les femmes se rassemblent pour prier Dieu à travers leur marabout puisqu' : 
elles ne se tournent pas vers la Mecque, elles, mais vers le corps du marabout, et elles se mettent sous sa protection directe, qui est encore, qui est toujours la protection de l'homme. Leurs yeux de femmes, leurs yeux doux et tristes, soulignés par deux bandeaux blancs, ne savent pas voir l'immatériel, ne connaissent que la créature. (16)

11 En lisant ce passage, l'idée qui nous vient à l'esprit, c'est l'âme féministe et compatissante de Maupassant jugé misogyne (Dahan, 1996), dans sa description de ces femmes prisonnières avec pitié et en critiquant par ailleurs la société patriarcale où c'est, comme il le note, "le mâle qui, vivant les nourrit, les défend, les soutient; c'est encore le mâle qui parlera d'elle à Dieu, après sa mort."(16). Nous avons donc l'impression que Maupassant s'apitoie sur le sort de ces femmes cloîtrées dans leur attachement au mythe de l'homme à la fois protecteur et bourreau. N'a-t-il pas dit aussi, en décrivant les maux des ces femmes soumises et endoctrinées par des idées fausses sur l'injustice divine à leur égard, que si elles ne viennent pas prier Dieu à la mosquée avec les hommes, c'est parce qu'elles n'oseraient pas :

lui raconter, tous les soucis, lui confier toutes les peines, lui demander tous les menus services, les menus consolations, les menus secours contre la famille, contre le mari, contre les enfants, dont ont besoin les cœurs des femmes. Il faut un intermédiaire plus humble entre lui si grand et elles si petites. (17)

Ce serait donc la raison pour laquelle, elles se dirigent vers ce marabout intermédiaire pour lui raconter «leurs affaires, leurs soucis, leurs disputes, les griefs contre le mari. » (17). Néanmoins, contrairement à "la mosquée sévère, nue, où Dieu est seul », ces femmes se retrouvent à la zaouiia pour causer, pour se décharger des peines de tous les jours. Et c'est à la zaouïa échappatoire aussi qu'elles se détournent de la souffrance de leur cœur par le bavardage mais aussi par le marivaudage :

C'est un boudoir, [décrit Maupassant la zaouïa], orné pour la prière par le goût enfantin de femmes sauvages. Souvent les galants viennent les voir en ce lieu, leur donner rendez-vous, leur dire quelques mots en secret. Des Européens, qui parlent l'arabe, nouent ici, parfois, des relations avec ces créatures enveloppées et lentes, dont on ne voit que le regard. (17)

Le voyageur se baladait en observateur et errait "au cour d'un espace et d'un peuple », (Mitterand, 1993 : 13). Du quai d'Alger où dans sa première cour, se trouve une petite pièce carrée dans laquelle "le cadi rend la justice » (8), à la mosquée, à la zaouïa, en traversant la ville arabe et dans l'un des labyrinthes de ses petites ruelles, se trouve « un long couloir sinueux et voûté » (9), où se trouvent les maisons closes. Dans ces lieux, on découvre «la liberté des mœurs, l'épanouissement en pleine rue, d'une prostitution innombrables, joyeuses... » (13). Un peu plus loin, se trouve une école «à côté de la fontaine où l'eau coule sous un arbre » (13). Bref, "Tout est là, [disait Maupassant], dans cette douce et paisible enceinte, la religion, la justice, l'instruction » (9). Alger apparaît, en outre, la ville des paradoxes où les mœurs changent, d'une religiosité exacerbée à un épanouissement et à une perversité discrets. Tout comme Tunis d'ailleurs...

14 En effet, après son voyage à Alger, Maupassant prend le chemin de fer pour arriver à Tunis. La première découverte visuelle cette fois-ci, ce sont les ruines des villes romaines dont parlait Flaubert dans Salammbô : en passant par « une suite de monts et de vallées désertes, où jadis s'élevaient des villes romaines » (19). Maupassant méditait sur les restes de Thagaste (Souk Ahras), la terre natale de saint Augustin, puis Thubursicum (Tboursok), Humidarum (Hadra mawt, du côté des frontières algériennes), ensuite sur 
Madaure (Madawrouch) jusqu'à Carthage. Selon Maupassant, " on ne pourrait guère énumérer les cités mortes, près desquelles on va passer jusqu'à Tunis. » (20).

D'une son trajet vers Tunis, Maupassant se focalise surtout sur la dimension sociologique de ce peuple multiracial. Ce qui a captivé le voyageur en quête des origines, c'est la diversité raciale qui existe à Tunis et en Tunisie. D'abord, à Tunis, il remarque qu'il y a trois populations qui partagent les quartiers de la ville : les Arabes, les juifs et les Français : "En vérité, Tunis n'est ni une ville française, ni une ville arabe, c'est une ville juive. C'est un des rares points du monde où le juif semble chez lui comme dans une patrie, où il est le maître presque ostensiblement, où il montre une assurance tranquille, bien qu'un peu tremblante encore » (21). Un métissage rare de races et de cultures retient le regard de Maupassant. Ainsi, il s'intéresse moins à la partie française de la ville qu'au brassage qui se dégage de cette panoplie de populations tunisiennes qui embellit le cadre spatial. Chacune son rythme, chacun ses coutumes, ses habits, son allure, ses tendances, sa personnalité ; mais elles sont toutes tunisiennes et incontestablement belles à voir :

Où sommes-nous ? Sur une terre arabe ou dans la capitale éblouissante d'Arlequin, d'un Arlequin qui s'est amusé à costumer son peuple avec une fantaisie étourdissante. Il a dû passer par Londres, par Paris, par Saint-Pétersbourg, ce costumier divin, qui revenu plein de dédain des pays du Nord, bariola ses sujets avec un goût sans défaillances et une imagination sans limites. (...) Oh! Pour ceuxlà, pour ces bons orientaux, ses Levantins métis de Turcs et d'Arabes, il a fait une collection de nuances si fines, si douces, si calmes, si tendres, si pâlies, si agonisantes et si harmonieuses, qu'une promenade au milieu d'elles est une longue caresse pour le regard. Voici des burnous de cachemire, (...). Et ces gebbas, ces vestes, ces gilets, ces haïks croisent, mêlent et superposent les plus fines colorations. (...) C'est un défilé de féerie. (21)

Maupassant tombe alors sous le charme de l'air hybride et original du Tunisien, et il est enivré voire apaisé par la fraîcheur de l'air de la Tunisie. Mise à part l'odeur « d'encens et d'aromates, un peu étourdissante » (27) qui flotte dans le souk, Maupassant, l'enfant du Nord et l'amoureux de la mer, trouve dans l'air de Tunis, "une ville saine, très saine " (29), le calmant naturel pour ses nerfs irrités et l'apaisement profond de son âme agitée: " L'air infect qu'on y respire, [disait Maupassant], est vivifiant et calmant, le plus apaisant, le plus doux aux nerfs surexcités que j'aie jamais respiré. » (29)

D'emblée, cet air exaltant et ensorcelant déclenche le désir de Maupassant et le guide vers la découverte inévitable de la vie nocturne de Tunis. Il pensait y trouver, comme à Alger, cet amalgame étonnant entre la vie pieuse et sérieuse de la journée et celle décontractée et érotique de la nuit. Cependant, selon ses dires « La ville arabe d'Alger est pleine d'agitation nocturne. » (34); or, "Dès que le soir vient, Tunis est mort. » (34). Dans cette ville, pour découvrir ses lieux nocturnes et discrets, a priori «morte» le soir, il faut prendre beaucoup de précautions, car tout se fait justement dans la discrétion. Les maisons closes n'apparaissent plus comme des lieux à risques mais comme des maisons publiques où tout se mêle : charme, art, épanouissement, érotisme et surtout vertu et péché :

(...) Elles sont parées comme pour une féerie, comme les princesses de Mille et une Nuit (...) deux autres femmes sont assises, deux prostitués (...). Elles chantent en tapant sur la darbouka avec leurs mains rougies par le henné, (...). Où sommes-nous, s'interroge Maupassant, Dans le temple de quelque religion barbare, ou dans une maison publique? Dans une maison publique? Oui, nous sommes dans une maison publique, et rien au monde ne m'a donné une sensation plus imprévue, plus fraîche, plus colorée que l'entrée dans cette longue pièce basse, où ces filles parées dirait-on 
pour un culte sacré attendant le caprice d'un de ces hommes graves qui semblent murmurer le Coran jusqu'au milieu des débauches. (38)

Ainsi s'arrête le voyage de Tunis. Maupassant part le 11 décembre vers Kairouan. Il décrit la route l'emmenant vers cette ville sainte comme étant déjà une «terre fanatique "(43). C'est alors par le truchement de cette terre en apparence fanatique que Maupassant entame sa comparaison avec le français colonisateur et avec l'Européen possesseur d'une terre fertile et d'un esprit plus civilisé et fort fier par rapport aux Arabes de l'Afrique maghrébine. En effet, dès son passage par le village de Grombalia, Maupassant évoque l'histoire andalouse de ce hameau qui fut fondé par un chef andalou musulman, Mohamed Gromabli, chassé d'Espagne par Isabelle la Catholique. En méditant les ruines byzantines, Maupassant s'arrête stupéfait devant la nouvelle mauvaise route principale artère de la Tunisie. Cette route qui commence de Grombalia en passant par Ennfidha et qui emmène à Kairouan était mal gérée par les ingénieurs français. "Celui d'Enfidaville a été construit deux fois (...). Celui d'Oued el Hammem est détruit pour la quatrième fois. (...) Seuls les vieux ponts arabes résistent à tout», (46) disait Maupassant. L'intervention française ne fait que détériorer la situation. Seules les indigènes savent comment y remédier.

19 La terre de ce pays est miraculeuse, selon Maupassant. Malgré l'aridité de son sol, la chaleur implacable de son soleil en été et malgré parfois le déluge de la pluie de son ciel hivernal, cette terre devient en printemps, écrivait-il :

Une prairie illimitée, avec des herbes montant aux épaules d'un homme et d'innombrables fleurs comme nous n'en voyons guère en nos jardins. (...) De Sahara sans un brin d'herbe, elle devient tout à coup presque en quelques jours, comme par miracle, une Normandie follement verte, une Normandie ivre de chaleur, jetant en ces moissons de telles poussés de sèves qu'elles sortent de terre, grandissent, jaunissent, et murissent à vue d'œil. Elle est cultivée de place en place, d'une façon très singulière, par les Arabes. (46)

20 Ainsi, l'Arabe toujours avec un grand 'A', séduit et envoute le Français européen. Cet Arabe est singulier même dans son comportement décrit comme un comportement «fataliste » par Maupassant. Il s'agit de sa sagesse nonchalante, qui consiste à laisser libre cours aux plantes qui poussent leurs racines dans la terre. L'Arabe suit la volonté de Dieu même dans la naissance d'une plante et évite de la déraciner, contrairement au Français ou à l'Européen qu'il tient avec acharnement à cultiver toute plante parasite et à la déraciner, violemment, farouchement. A ce propos Maupassant écrit :

On retrouve bien, dans cette indifférence tranquille, dans ce respect pour la plante poussée sur la terre de Dieu, l'âme fataliste de l'Oriental. Si elle a grandi là, cette plante, c'est que le Maître l'a voulu, sans doute. (...) Chez nous, le paysan, rageur, jaloux de la terre plus que de sa femme, se jetterait, la pioche aux mains, sur l'ennemi poussé chez lui, sans repos jusqu'à ce qu'il l'eût vaincu, il frapperait, avec de grands gestes de bûcheron, la racine tenace enfoncée au sol. (50)

21 Nous remarquons dans ce passage, la métaphore filée de la plante qui apparaît telle une intruse alors qu'à la base c'est elle l'indigène, la propriétaire des lieux par la volonté de Dieu. Elle est de toutes les façons l'originaire dans cette terre; l'Arabe de son côté respecte ses origines et veille à ce que la volonté de Dieu soit protégée ; or, l'Européen la prend carrément pour un concurrent voire un ennemi et s'en prend avec acharnement même à ses racines. L'un (l'Arabe) respecte ses origines, de la plante, et l'autre (le colonisateur) tient à les éliminer. 

lui, la décision des Français de la reconstruction d'une nouvelle route, cette fois-ci celle de Bou-Ficha. Avec son chauffeur, ils apercevaient avant l'entrée à cette Provence « une armée d'ouvriers de toutes races occupés à remplacer ce chemin passable par une voie française, c'est-à-dire par un chapelet de danger. ». (53). Ce qui a fasciné encore une fois le voyageur c'est la diversité des nationalités de ces ouvriers, du «nègre Lippu », à «l'Arabe au fin profil », à "l'Espagnole poilu », au « Marocain », et aussi au " Maure », au «Maltais », au «terrassier français égaré » au «Grecs » et au «Turcs». Tout le monde existe en Tunisie et toutes les origines cohabitent.

Après une nuit de repos chez M. Moreau, l'intendant de Bou-Ficha, le voyageur reprend sa route vers Kairouan. En longeant de loin la côte du petit village de Hergla, Maupassant s'arrête épaté par la couleur rose de l'aurore. Sa description de cette lumière matinale a pris plus qu'une page dans ce bref récit. Après tant de mots et de phrases, l'écrivain conclut être incapable de décrire ce magique paysage rare : «Ce que j'ai vu ce matin là, en quelques minutes, je ne saurais, avec des verbes, des mots et des adjectifs, le faire voir. » (55). Après s'être enchanté de cette vue, Maupassant est émerveillé par ce qu'il aperçoit de loin, dans son parcours impressionnant de voyage: les ruines de l'amphithéâtre d'Eljam et la grandeur de son histoire épique. En avançant son trajet, le voyageur croise au fur et à mesure des petits villages et leurs environs ; ce qui l'a attiré le plus c'est surtout ces villageois et campagnardes vêtus différemment des citadins. Ils sont typés malgré la diversité de leurs habits berbères et arabes. ${ }^{3}$ Le comble pour Maupassant, c'est que plus il observe ces belles créatures légendaires plus il arrive à mieux cerner l'histoire et à mieux comprendre la Bible :

Dans ce pays, on apprend par ses yeux ce qu'est l'histoire et surtout ce que fut 1 Bible. On comprend que les patriarches et que tous les personnages légendaires, si grands dans les livres, si imposants dans notre imagination, furent de pauvres hommes qui erraient à travers les peuplades primitives, comme errent ces Arabes graves et simples, pleins encore de l'âme antique et vêtus de costume antique. Les patriarches ont eu seulement des poètes historiens pour chanter leur vie. (55)

Le spectacle légendaire et mythique passe devant les yeux hypnotisés de Maupassant tout au long de son passage. Au pied d'un olivier ou à côté d'un cactus, il aperçoit la Vierge Marie un peu courbée portant dans un burnous gris de poussière, l'Enfant Jésus. Plus loin à chaque puits il voit, Rebecca, la femme d'Isaac et la mère de Jacob :

(...) habillée d'une robe en laine bleue, superbement drapée, porte aux chevilles des anneaux d'argent et, sur la poitrine, un collier de plaques du même métal, unies par des chaînettes. (...) C'est bien la fille de la Bible. (...) En Algérie et dans le Sahara algérien, toutes les femmes, celles des villes comme celles des tribus, sont vêtues en blanc. En Tunisie, au contraire, celles des cités sont enveloppées de la tête aux pieds en des voiles de mousseline noire (...), et celles des campagnes sont habillées avec des robes gros bleu d'un gracieux et grand effet, qui leur donne une allure encore plus biblique. (58)

À travers cette description, Maupassant évoque l'authenticité des véritables origines historiques et religieuses que ni les livres sacrés ni les Encyclopédies de tous genres n'arrivent simplement à traduire et prodigieusement à illustrer (Biaggi et Baland, 1993).

Le 15 décembre, Maupassant arrive à destination. Voici enfin Kairouan, la ville sainte de l'Afrique. Ce qui a marqué à première vue le voyageur c'est le choix de 'Sidi Okba' comme le nomme-t-il tout les kairouanais, de cette cité à géographie " aride et désolée " pour installer son armée et fonder la troisième ville sainte après La Mecque et 
Jérusalem. Cette ville possède le plus grand nombre des Kobbas, des Zaouis en Tunisie à part la grande mosquée de Sidi Okba et la mosquée du Barbier du Prophète et l'un de ses Apôtres, Sidi Essahbi. Maupassant comprend la grande quantité de ces lieux de prières et de vénération de Dieu par la certitude des croyants qui pensent que «sept pèlerinages à Kairouan valent un pèlerinage à La Mecque. » (71).

Après avoir observé les ruelles de la Medina et ses commerçants typiquement orientaux, le puits de » Barrouta » où le chameau tourne au rond dans la coupole pour monter l'eau, le voyageur passionné prend son bain dans un Hammam et découvre, dès lors, la vapeur de l'eau chaude et naturelle de cette enceinte mystérieuse et les massages apaisants et rafraîchissants des spécialistes en gommage du corps. À travers ce petit plaisir, Maupassant ressent l'allégresse d'un fabuleux sentiment d'un état de bien-être qui manquait aussi bien son esprit que son corps depuis quelque temps :

Or, après toutes les opérations du massage, quand nous revenons au grand air, une ivresse de joie nous étourdit, car le soleil levé illumine les rues et nous montre, blanche comme toutes les villes arabes, mais plus sauvage, plus durement caractérisées, plus marquée de fanatisme, saisissante de pauvreté visible, de noblesse misérable et hautaine, Kairouan la sainte. (73)

Le voyageur se rend maintenant à la mosquée Djama-Kebir de Sidi-Okba. Après tant de voyages effectués en Europe, le connaisseur dans la matière déclare son admiration pour l'un des meilleurs édifices religieux jamais vus. À part le Mont Saint-Michel, SaintMarc de Venise et la chapelle Palatine de Palerme, la grandeur du Djama-Kebir se résume en ce que ce petit peuple avec des moyens minimes a pu construire avec tant d'art et d'ingénierie. Maupassant décrit son émotion « inattendue et foudroyante » lors de la méditation de «ce barbare et surprenant monument », et dit :

Ici c'est autre chose. Un peuple fanatique, errant, à peine capable de construire des murs, venu sur une terre couverte de ruines (...), y ramassa partout ce qui lui parut de plus beau (...) avec ces débris du même style et de même ordre, éleva, mû par une inspiration sublime, une demeure à son Dieu (...). Le regard s'arrête, se perd dans cet emmêlement profond de minces piliers ronds d'une élégance irréprochable, dont toutes les nuances se mêlent et s'harmonisent, et dont les chapiteaux byzantins, de l'école africaine et de l'école orientale, sont d'un travail rare et d'une diversité infinie. (75)

Le point qui a saisi, surtout, Maupassant c'est la dimension basique et horizontale qui caractérise cette mosquée, contrairement à la dimension élevée et hautaine caractérisant ainsi les cathédrales gothiques. N'avait-il pas dit, au début de son récit lors de sa visite d'une mosquée à Alger que :

Tout est simple, tout est nu, tout est blanc, tout est doux, tout est paisible en ces asiles de foi, si différents de nos églises décoratives, agitées, quand elles sont pleines, par le bruit des offices, le mouvement des assistants, la pompe des cérémonies, les chants sacrés, et, quand elles sont vides, devenues si tristes, si douloureuses, qu'elles serrent le cœur, qu'elles ont l'air d'une chambre de mourant, de la froide chambre de pierre où le Crucifié agonise encore. (9)

À Kairouan, Maupassant continue sa description de sa mosquée par opposition aux églises et dit :

Dans nos cathédrales gothiques, le grand effet est obtenu par la disproportion voulue de l'élévation avec la largeur. Ici, au contraire, l'harmonie unique de ce temple bas vient de la proportion et du nombre de ces fûts légers qui portent l'édifice, l'emplissent, le peuplent, le font ce qu'il est, créent sa grâce et sa grandeur. (...) Cela est vaste comme un monde. Le Dieu qui a inspiré cette œuvre d'art superbe est bien celui qui dicta le Coran, non point celui des Evangiles. Sa 
morale ingénieuse s'étend plus qu'elle ne s'élève, nous étonne par sa propagation plus qu'elle ne nous frappe par sa hauteur. (76) l'attachement de l'Arabe à la notion des racines qui doivent être les bases d'une construction solide et harmonieuse, contrairement à l'Européen qui s'intéresse plus à la hauteur sans se fier vraiment à la base des origines. Par ailleurs, le voyageur découvre un autre genre de mosquée moins imposante mais aussi gracieuse de toutes les mosquées croisées auparavant. Il s'agit de la mosquée du Barbier du Prophète, elle est selon ses dires : « la plus colorée, la plus coquette des mosquées, et le plus parfait échantillon de l'art décoratif arabe que j'aie vu. » (78). La visite de ces lieux mystiques et féeriques en même temps, se clôture par la rencontre de Maupassant avec un cheik duquel il a retenu ces quelques phrases:

Prier et jeûner dans la solitude et n'avoir aucune compassion dans le cœur, cela s'appelle dans la bonne voie, de l'hypocrisie. L'amour est le degré le plus complet de la perfection. Il y a quatre sortes d'amour : l'amour par l'intelligence, l'amour par le cœur, l'amour par l'âme, l'amour mystérieux. (86)

Epaté et abasourdi par une telle profondeur, le grand écrivain se demande après avoir entendu ce cheik arabe : "Qui donc a jamais défini l'amour d'une manière plus complète, plus subtile et plus belle?». (86)

Enrichi, confus et émerveillé par toutes ces découvertes et rencontres, Maupassant quitte Kairouan le 16 décembre vers Sousse. Dès l'apparition de Sousse, l'âme enfantine et l'esprit normand et amoureux de l'Océan de Maupassant se déclenchent. Il a vu enfin une de ses muses refoulées, la muraille qui emmène et longe la mer. Excité et avec un rythme saccadé et une énumération remarquable, l'écrivain montre sa joie de retrouver ses propres racines dans sa quête des origines de l'autrui, dans l'ailleurs :

Voici Sousse.

Mais, je l'ai vue, cette ville! Oui, oui j'ai eu cette vision lumineuse autrefois, dans ma toute jeune vie, au collège, quand $j$ 'apprenais, dans ma toute jeune vie, au collège, quand j'apprenais les croisades dans l'Histoire de France de Burette. Oh! Je la connais depuis longtemps! (...) Rien que pour voir Sousse, on devrait faire ce long voyage. (...). (91)

Maupassant finit son voyage par des retrouvailles qui suscitent les réminiscences de son enfance et qui lui rappellent ses origines. Il découvre tout au long de son parcours de Tunis à Kairouan et qui finit par Sousse, trois grandes vérités : la première consiste à lui montrer que la diversité des races et des genres ne font qu'enrichir et embellir un peuple, la deuxième consiste à lui apprendre que le fanatisme n'est pas un choix, c'est une culture qui pourrait être pacifique et étonnante dans un cadre géographique austère ; la troisième vérité, c'est que malgré les voyages et les tentatives de s'échapper de son moi le plus profond, personne n'est capable de fuir ses origines et de se détacher de ses racines. Un simple regard, un fugitif souvenir, une rencontre imprévue susciteraient tôt ou tard, ici ou ailleurs, les âmes sensibles et fidèles.

Avant de finir, nous ne pouvons pas passer à côté d'un détail très révélateur voire précurseur dans ce récit. Lors de sa visite dans un hôpital psychiatrique à Tunis, Maupassant décrit ce qu'il a vu et écrit :

Je voulus passer en revue ces déments effrayants et admirables en leur costume oriental, plus curieux et moins émouvants peut-être, à force d'être étranges, que nos pauvres fous d'Europe. (...) Puis en voici un vieux qui rit et nous crie, en dansant comme un ours : 
- Fous, fous, nous sommes tous fous, moi, toi, le médecin, le gardien, le bey, tous, tous fous! (...). Il nous désigne l'un après l'autre, et rit, car il est sûr que nous sommes fous, lui, ce fou, et il répète :

- Oui, oui, toi, toi, toi, tu es fou ! (31-32-33)

Et on croit sentir pénétrer en son âme un souffle de déraison, une émanation contagieuse et terrifiante de ce dément malfaisant.

En 1888 Guy de Maupassant assiste en Tunisie à cette scène du fou qui le désigne comme un fou à son tour. Cinq ans après en 1893, l'écrivain quitte la vie dans un asile psychiatrique de la région parisienne en ayant les mêmes symptômes et en faisant les pareils gestes du fou de Tunis. Un signe? Une prémonition? Ou une anticipation de son destin final ? Ce qui est indéniable, c'est que dans l'hôpital tunisien, Maupassant n'était pas uniquement en face de ce fou Arabe, mais surtout il était en face de l'origine même de son «mal d'être », qui n'est finalement que son destin (Borel, 1927).

\section{BIBLIOGRAPHIE}

BIAGGI, Vladimir, Au salon (chroniques picturales), Balland, Paris, 1993.

BOREL, Pierre., Le destin de Guy de Maupassant, Les Editions de France, Paris, 1927.

BURY, Marianne, « Maupassant pessimiste ? ", in Romantisme, $\mathrm{n}^{\circ}$ 61, 1988, pp. 75-83.

CZYBA, Luce , Ecrire au XIX ${ }^{\circ}$ siècle, Les Belles Lettres, Paris, 1998.

DAHAN, Philippe, Maupassant et les femmes, Editeurs Bertout-la mémoire, Normandie, 1996.

Delaisement, Gérard, Guy de Maupassant, Chroniques, I, II, Rive droite, Paris, 2004.

JACQUES, Réda, Album Maupassant, Gallimard, Paris, 1987.

LECLERC, Yvan., Choses et autres : choix de chroniques littéraires et mondaines (1876-1890), Le Livre de poche, 2011.

MAUPASSANT, Guy de, Chroniques et récit de voyage, Bruxelles, Complexe, coll, « Le regard littéraire », 5 vol, 1993.

MAUPASSANT, Guy de, De Tunis à Kairouan, Editions Complexes, Bruxelles, 1993.

MORAND, Paul, Vie de Guy de Maupassant, Flammarion, 1942.

PASQUET, Martin Maupassant : Biographie, étude de l'œuvre, Albin Michel, Paris, 1993.

SALEM, Jean, Philosophie de Maupassant, Ellipses, Paris, 2000.

SATIAT, Nadine, Maupassant, Flammarion, Paris, 2003.

schmidT, Albert Marie, Maupassant, éd. Ecrivains de toujours, Seuil, Paris, 1990.

TVERDOTA, György, Ecrire le voyage (textes réunis), Presse de la Sorbonne Nouvelle, Paris, 1994.

WAUTERS, Eric, Tunis, Carthage, l'orient sous le regard de l'occident du temps des Lumières à la jeunesse de Falubert, édi, Publications de l'Université de Rouen, 1999. 


\section{NOTES}

1. Luce Czyba, écrit dans ce contexte : "Il convient de préciser la nature et la fonction de ce modèle aristocratique. Il révèle tout d'abord l'ambivalence du bourgeois à l'égard de l'argent : "pour mener la vie élégante", il faut cent mille livres de rente (...). A ce prix seulement on fait partie de la "société" qui est "le sommet de la civilisation" et à partir de laquelle se définissent les critères de la beauté, du goût, de l'esprit et de l'élégance... », Luce CZYBA, Ecrire au XIX siècle, Les Belles Lettres, 1998, p. 62.

2. Textes réunis par Eric Wauters, Tunis, Carthage, l'Orient sous le regard de l'Occident du temps des Lumières à la jeunesse de Flaubert, éd. Publications de l'Université de Rouen, p. 126, 1999.

3. Voir, Chronique et récit de voyage, Bruxelles, Complexe, coll. Le Regard littéraire, 1993, 5 vol.

\section{RÉSUMÉS}

«Le voyage est une espèce de porte par où l'on sort de la réalité connue pour repénétrer dans une réalité inexplorée qui semble un rêve. ». Ainsi commence De Tunis à Kairouan publié en plusieurs parties dans Le Gaulois du 11 décembre 1888 et dans La Revue des Deux Mondes en 1889. De Tunis à Kairouan est une chronique relatant un parcours de voyage que Maupassant a effectué (cinq ans avant sa mort) dans les deux colonies maghrébines, l'Algérie et la Tunisie. Dans ce récit de voyage, Maupassant s'évade et erre pour oublier, mais aussi pour tenter de «se déraciner " en explorant une terre inconnue et des origines vierges de tout sens d'artificiel, d'absurdité, de férocités propres au monde européen et à la société française. Maupassant se dirige vers un univers où tout est différent, naturel, simple, farouche, ethnique, culturel, historique, religieux, mystique, profane, vertueux, authentique, paradoxal, divers, mystérieux, beau et fascinant, bref, un monde où tout est berbère, arabe et nord africain.

"A journey is a kind of door through which we go out of the known reality to re-enter an unexplored reality that seems a dream." So begins From Tunis to Kairouan published in several parts in Le Gaulois of December $11^{\text {th }}, 1888$ and in La Revue des Deux Mondes in 1889. From Tunis to Kairouan is a chronicle, relating a journey that Maupassant made (five years before his death) in two Maghreb colonies, Algeria and Tunisia. In this travel story, Maupassant escapes and wanders to forget. But also to try to "root out" by exploring an unknown land and origins virgin of all sense of artifice, absurdity, ferocity peculiar to the European world and to the French society. Maupassant moves towards a universe where everything is different, natural, simple, fierce, ethnic, cultural, historical, religious, mystical, profane, virtuous, authentic, paradoxical, diverse, mysterious, beautiful and fascinating ; in brief, where everything is Berber, Arabic and North African. 
INDEX

Mots-clés : Maupassant, voyage, Algérie-Tunisie, quête, origines

$$
\text { موباسان, الرحلة, الجزائر- تونس, البحث, الأصولفهرس الكلمات المفتاحية: }
$$

Keywords : Maupassant, travel, Algéria-Tunisia, quest, origins

\section{AUTEUR}

DORRA BARHOUMI

Université de Kairouan 